\title{
Conditioned pain modulation dampens the thermal grill illusion
}

\author{
D.E. Harper ${ }^{1,2}$, M. Hollins ${ }^{1}$ \\ 1 Department of Psychology and Neuroscience, University of North Carolina at Chapel Hill, USA \\ 2 Department of Anesthesiology, Chronic Pain and Fatigue Research Center, University of Michigan, Ann Arbor, USA
}

\begin{abstract}
Correspondence
Daniel E. Harper

E-mail: harperd@med.umich.edu

Funding sources

This research was supported by a King Research Excellence Award (to DEH). DEH is currently supported by an institutional grant from NIH (K12-DE023574) at the University of Michigan.
\end{abstract}

Conflicts of interest

None declared.

Accepted for publication

21 April 2017

doi:10.1002/ejp.1060

\begin{abstract}
Background: The thermal grill illusion (TGI) refers to the perception of burning heat and often pain that arises from simultaneous cutaneous application of innocuous warm and cool stimuli. This study utilized conditioned pain modulation (CPM) to help elucidate the TGI's underlying neural mechanisms, including the debated role of ascending nociceptive signals in generating the illusion.

Methods: To trigger CPM, subjects placed the left hand in noxious cold $\left(6{ }^{\circ} \mathrm{C}\right)$ water before placing the right volar forearm onto a thermal grill. Lower pain and unpleasantness ratings of the grill in this CPM run compared to those in a control run (i.e. $33^{\circ} \mathrm{C}$ water) were taken as evidence of CPM. To determine whether CPM reduces noxious heat pain and illusory heat pain equally, an experimental group of subjects rated pain and unpleasantness of a grill consisting of innocuous alternating warm $\left(42{ }^{\circ} \mathrm{C}\right)$ and cool $\left(18{ }^{\circ} \mathrm{C}\right)$ bars, while a control group rated a grill with all bars controlled to a noxious temperature $\left(45^{\circ} \mathrm{C}\right)$.
\end{abstract}

Results: CPM produced significant and comparable reductions in pain, unpleasantness and perceived heat of both noxious heat and the TGI.

Conclusions: This result suggests that the TGI results from signals in nociceptive dorsal horn convergent neurons, since CPM involves descending inhibition with high selectivity for this neuronal population. More broadly, CPM's ability to produce a shift in perceived thermal sensation of both noxious heat and the TGI from 'hot' to 'warm' implies that nociceptive signals generated by a cutaneous stimulus can contribute to its perceived thermal intensity.

Significance: Conditioned pain modulation reduces the perceived painfulness, unpleasantness and heat of the thermal grill illusion and noxious heat similarly. The results have important theoretical implications for both types of pain.

\section{Introduction}

An early theory of Thunberg's (1896) thermal grill illusion (TGI) was based on the then-recent discovery that noxious heat not only activates warm spots on the skin but also paradoxically activates cold spots. The thermal grill, which activates warm spots with warmth and cold spots with cool, was thought to mimic the dual activity produced by noxious heat (Alrutz, 1898). Thus, Alrutz reasoned that the TGI, and more generally the qualitative perception of heat, results from experiencing two innocuous sensations simultaneously.

Other theories of the TGI suggest that it involves the nociceptive system. Craig and Bushnell (1994) posited (1) that the grill's cool bars activate C polymodal nociceptors, which project to convergent dorsal horn neurons that code for pain; and (2) that the warm bars of the grill inhibit activity in pain-inhibitory dorsal horn COOL neurons (i.e. those that transmit innocuous cool sensations) but not 
convergent nociceptive neurons. In other words, the TGI results from a relative enhancement of nociceptive signals that are masked under normal conditions (Craig and Bushnell, 1994). More recent psychophysical evidence also supports the idea that the cool bars transmit the pain signals underlying the TGI (Harper and Hollins, 2014). Alternatively, the signals from innocuous warm and cool might integrate into the nociceptive system by converging onto dorsal horn wide dynamic range (WDR) neurons (Green, 2002; Bouhassira et al., 2005). Under either theory, the TGI involves activity in dorsal horn nociceptive neurons.

Although many researchers have found that the TGI is painful to a majority of participants, others have debated the painfulness of the TGI and the involvement of the nociceptive system in generating the illusion (Fruhstorfer et al., 2003; Bach et al., 2011). Furthermore, a recent study reported that the TGI is not painful in cats (Boettger et al., 2016), calling into question the translatability of Craig and Bushnell's (1994) physiological findings.

To empirically test the nociceptive system's role in producing the TGI in humans, conditioned pain modulation (CPM) served as an analytical tool. CPM involves measuring changes in the pain of a noxious test stimulus due to a noxious conditioning stimulus, which is applied to a remote body location. CPM's primary physiological mechanism, diffuse noxious inhibitory controls (DNIC), selectively inhibits dorsal horn convergent nociceptive neurons (Le Bars et al., 1979). CPM may also recruit additional descending inhibitory projections from other regions, including the anterior cingulate cortex and the periaqueductal grey (Piche et al., 2009; Sprenger et al., 2011; Bogdanov et al., 2015; Youssef et al., 2016a,b); however, it is generally accepted that the majority of CPM's inhibitory effects result from attenuation at the spinal level and that descending inhibition selectively attenuates nociceptive signals (Waters and Lumb, 1997; Heinricher et al., 2009; Leith et al., 2010).

The TGI is produced by combining stimuli that are individually described as innocuous. Because CPM reduces nociceptive but not innocuous signals at the level of the spinal cord, similar reductions in the painfulness of nociceptive heat and the TGI by CPM would provide good evidence that the TGI results from spinally mediated nociceptive signals.

\section{Methods}

\subsection{Participants}

Subjects were recruited from a posting on the UNC$\mathrm{CH}$ Psychology Department's participant pool website. Written informed consent was obtained from each participant prior to the start of the experimental session. Upon completion of the experiments, participants were compensated with credit towards the Introductory Psychology research participation requirement. All procedures were approved by the University's Institutional Review Board.

Thirty-seven healthy undergraduate students participated in the main experiment, which tested the effects of CPM on the TGI and noxious heat. Subjects were randomly assigned to either the Thermal Grill (TG) group ( $n=18 ; 6$ males) or the Noxious Heat (NH) group $(n=19 ; 7$ males). Ages ranged from 18 to $22($ mean $=19.1 ; S D=1.2)$ and from 18 to $20($ mean $=19.1 ; \mathrm{SD}=0.9)$ in the $\mathrm{TG}$ and $\mathrm{NH}$ groups, respectively.

Twenty-four additional subjects ( 7 males) were enrolled in a separate component temperatures experiment, in which the warm and cool temperatures used to produce the TGI in the main experiment were applied individually (and without CPM). Their ages ranged from 18 to 21 years $($ mean $=18.6$; $\mathrm{SD}=0.8)$. None had participated in the main experiment.

\subsection{Design of main experiment}

Subjects assigned to the TG group were exposed to a grill consisting of interlacing warm $\left(42{ }^{\circ} \mathrm{C}\right)$ and cool $\left(18^{\circ} \mathrm{C}\right)$ bars to produce the TGI. Subjects in the $\mathrm{NH}$ group were exposed to bars that were all heated to a noxious temperature $\left(45^{\circ} \mathrm{C}\right)$, to elicit nociceptive heat pain. Participants in both groups were exposed to their respective grill stimulus on the right volar forearm twice, in separate runs. One exposure (Control run) took place while the left hand was immersed in a neutral $\left(33^{\circ} \mathrm{C}\right)$ water bath and the other (CPM run) while it was positioned in a painfully cold $\left(6^{\circ} \mathrm{C}\right)$ bath. The order of the two runs was counterbalanced within each group.

Thus, the between-subjects factor was the type of pain that subjects experienced (NH vs. TG), and the within-subjects factor was whether the left hand was in painfully cold or neutral water (CPM vs. Control) while the grill was presented. This design permitted analysis of three effects: (1) pain experience of noxious heat versus the thermal grill; (2) amount of pain reduction by CPM; and (3) assessment of CPM's relative ability to reduce the two types of pain.

We chose to have participants immerse their left hand in a neutral water bath for our baseline measure of the test pain, instead of having them do nothing for the baseline (other than feel the test stimulus) as is commonly done in CPM paradigms. 
This design minimized the potential for confounding effects on the test stimulus like distraction from placing a hand in water, giving conditioning stimulus ratings before presentation of the test stimulus, or any number of differences across conditions that could have added error to the measurement. By making the two runs as similar as possible for each subject, we intended to focus in on the CPM effects that are most directly related to nociceptive stimulation. Furthermore, it is known that weak, innocuous thermal stimulation is not sufficient to condition pain (Willer et al., 1984; Granot et al., 2008; Nir et al., 2011).

\subsection{Design of component temperatures experiment}

The experiment using separate component temperatures was implemented to measure the sensations associated with separate presentations of the warm and cool temperatures that were used to induce TGI. Subjects participated in two runs, one with all of the bars at the warm temperature and a second with all of the bars at the cool temperature. The order of these two runs was counterbalanced. No water bath was used in this experiment.

\subsection{Apparatus and materials}

\subsubsection{Thermal grill}

The thermal grill apparatus consisted of 12 copper tubes (length $33 \mathrm{~cm}$; diameter $1 \mathrm{~cm}$; thickness $0.4 \mathrm{~mm}$ ) that were secured with twine onto the top of a plastic holder (Fig. 1A). Each bar rested in a trough ( $1.25 \mathrm{~cm}$ wide and $0.5 \mathrm{~cm}$ deep) and thus was separated from its neighbour(s) by $0.5 \mathrm{~cm}$. In order to gain thermal control over the bars, two sets of plastic tubing through which water could be circulated were connected to the ends of the bars. Each intake was connected to a thermally insulated 19-L tank that was positioned on a shelf $0.65 \mathrm{~m}$ above the tabletop. Before an experimental run, the experimenter filled each tank with $15 \mathrm{~L}$ of water. Water was allowed to flow through the apparatus for 1 min to ensure temperature stabilization before each run began.

Two thermistor probes (YSI 400 series) were attached to small sections of copper tubing that were inserted into the flow lines near the grill to record bar-surface temperatures during experiments.

Based on preliminary testing, we determined warm and cool temperatures that produced a moderately intense TGI but were not perceived to be painful on their own. These bar temperatures were $42{ }^{\circ} \mathrm{C}$ and $18^{\circ} \mathrm{C}$.

\subsubsection{Water bath}

To measure the effects of CPM in the main experiment, the left hand was lowered into a water bath before application of the grill. The conditioning stimulus for inducing CPM consisted of an 11-L plastic cooler $\left(23.5 \mathrm{~cm}^{3}\right)$, which was filled with $10 \mathrm{~L}$ of water before the start of each run. An aquarium pump was used to circulate water and ensure stable temperatures surrounding the hand. A plastic grate divided the interior of the cooler into two chambers, in order to separate the subject's hand from the pump and any ice cubes. A thermometer was used to measure the temperature of the water and control it (by adding ice or warm water) to the desired temperature before a given run. The temperature of the water was painfully cold $\left(M=6.1{ }^{\circ} \mathrm{C} ; \mathrm{SD}=0.08\right)$ during the $\mathrm{CPM}$ run and was neutral $\left(M=32.7{ }^{\circ} \mathrm{C}\right.$; $\mathrm{SD}=0.26$ ) during the Control run.

\subsubsection{Questionnaires}

Before participating in the experimental runs, all subjects filled out a demographics questionnaire (age, sex, race and handedness).

Immediately following each experimental run, $\mathrm{NH}$ and TG subjects were provided with a sensation questionnaire that asked subjects to characterize sensations associated with the grill by circling words (as many as applied) from a list of descriptors: neutral, cool, cold, warm, hot, burning, stinging, sharp and aching. This questionnaire was used to assess qualitative differences in the pain and thermal sensations experienced under different experimental conditions.

Subjects in the component temperatures experiment filled out a different sensation questionnaire following each exposure to the grill, which in their case was either warm or cool. Descriptors for this questionnaire focused on temperature, pleasantness and pain and were neutral, cool, cold, warm, hot, painful, comfortable, pleasant and unpleasant. Subjects were instructed to indicate any that applied to their experience.

\subsection{Procedure}

\subsubsection{Main experiment}

The experimenters filled the tanks supplying the grill and the water bath with water and controlled them to the desired temperatures prior to the subject's arrival to the lab.

After giving informed consent and filling out the demographics questionnaire, the subject was trained to use a 0-100 scale to rate sensations. 

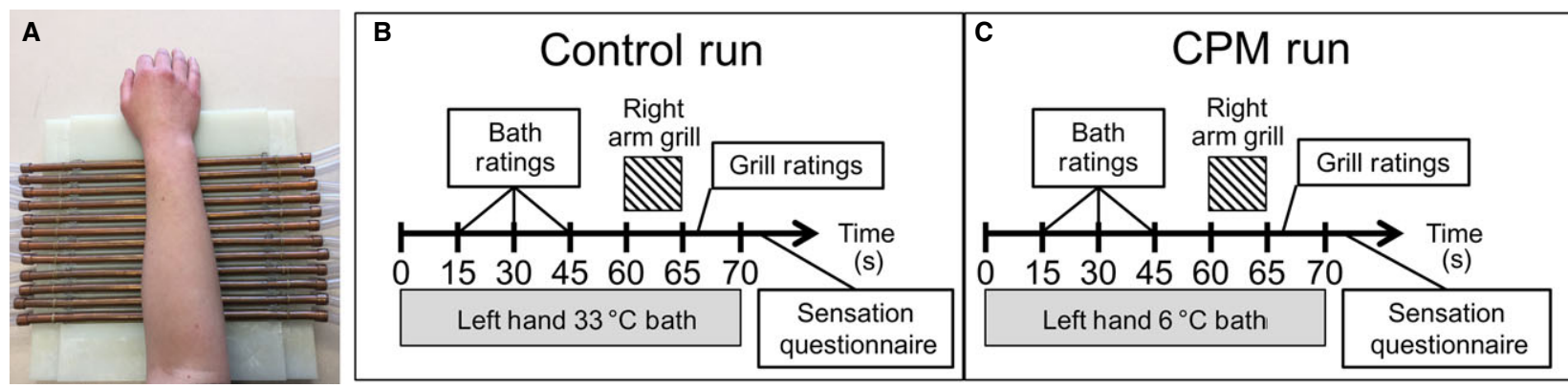

Figure 1 The thermal grill apparatus and experimental protocol. (A) The thermal grill was composed of 12 cylindrical copper bars arranged parallel to one another and held in place on a plastic base. The subject placed his or her right volar forearm onto the grill perpendicular to the long axis of the bars, as shown. For those in the noxious heat group, the grill was controlled to $45^{\circ} \mathrm{C}$, while for the thermal grill group, bars at $18{ }^{\circ} \mathrm{C}$ and $42{ }^{\circ} \mathrm{C}$ were interlaced to produce the TGI. In the component temperatures experiment, all of the bars were controlled to $18{ }^{\circ} \mathrm{C}$ in one run and all were controlled to $42^{\circ} \mathrm{C}$ in the other run. (B) The timing of events in a Control run is shown. The only difference between the Control run and the (C) CPM run was the temperature of the water bath. Each participant in the main experiment felt their group's respective grill twice, once with and once without the noxious conditioning stimulus. The order of these two runs was counterbalanced. Note that the timeline increments are not to scale.

Following this training, the subject was seated at a table on which the thermal grill apparatus rested, and the procedures were explained in detail. The water bath, for left hand immersion, was positioned on a chair to the left of the subject. The run began when the subject placed his or her left hand into the water bath up to the wrist. The subject was prompted for verbal ratings of pain intensity of the water bath on a 0-100 scale, where 0 meant 'no pain' and 100 meant 'the most intense pain imaginable', every $15 \mathrm{~s}$ for the first $45 \mathrm{~s}$ of the run. One minute into the run, the subject was told to place the volar surface of his or her right forearm onto the grill apparatus, and $5 \mathrm{~s}$ later was told to remove it. The subject was then prompted for verbal ratings of grill pain intensity (0-100 scale) and unpleasantness (0-100 scale from 'not at all unpleasant' to 'the most unpleasant sensation imaginable'). After grill ratings were obtained, the subject removed his or her hand from the water bath and dried it off with paper towels. The sensation questionnaire for that run was administered immediately afterwards (Fig. 1B). Thus, we obtained numerical ratings of both pain and unpleasantness of the grill along with indications (i.e. yes/no responses) of whether participants experienced certain qualitative aspects associated with thermal stimulation.

The subject took a 25-min break between runs to minimize any lingering effects (e.g. sensitization or habituation) of exposure to the thermal stimuli during the first run and to allow the experimenters to prepare the stimuli for the second run. The grill temperatures for the second run were the same as for the first (i.e. $42{ }^{\circ} \mathrm{C} / 18{ }^{\circ} \mathrm{C}$ for subjects in the TG group or $45^{\circ} \mathrm{C} / 45^{\circ} \mathrm{C}$ for those in the NH group). The temperature of the conditioning water bath was adjusted to either $6{ }^{\circ} \mathrm{C}$, if the subject underwent the Control run first, or $33^{\circ} \mathrm{C}$, if the first was a CPM run. Procedures were otherwise identical in the two runs (Fig. 1C). Following administration of the sensation questionnaire for the second run, the subject was debriefed and awarded credit for his or her participation.

Bar-surface temperature was recorded in the seconds before the subject placed his or her forearm on the grill to ensure that the applied temperatures were within the desired range. For the $\mathrm{NH}$ group, the average bar temperatures $\left({ }^{\circ} \mathrm{C}\right)$ of sets 1 and 2 were $44.75(\mathrm{SD}=0.39)$ and $44.87(0.41)$ during the Control run and 44.71 (0.48) and 44.87 (0.41) during the CPM run, respectively. For the TG group, the average bar temperatures of the warm and cool sets were $41.96(0.37)$ and $18.18(0.15)$ during the Control run and $42.01(0.39)$ and $18.22(0.17)$ during the CPM run, respectively.

\subsubsection{Component temperatures experiment}

In order to determine whether the warm and cool bars were perceived as innocuous on their own in naive subjects, 24 additional participants were enrolled in an experiment in which they felt the grill with all bars controlled to either $18{ }^{\circ} \mathrm{C}$ or $42^{\circ} \mathrm{C}$. Prior to each run, both tanks supplying the grill were filled with water that was either warm (for the warm run) or cool (for the cool run).

After giving informed consent and filling out the demographics form, the subject was seated at the experimental table on which the grill rested. The 
valves for the water were opened and water was allowed to flow through the bars for at least $1 \mathrm{~min}$ to allow ample time for bar temperature stabilization. The experimenter then told the subject to put his or her right forearm onto the grill and indicated (after $5 \mathrm{~s}$ ) when to remove it. The sensation questionnaire was administered immediately thereafter.

The subject took a 20-min break before participating in a second run, in which he or she was exposed to the temperature (warm or cool) not used during the first run. The procedures were identical. Run order was randomized prior to the beginning of experimentation, subject to the constraint that half of the subjects underwent the warm, and the other half the cool, run first.

Data from four runs (one cool and three warm) in which the temperatures of one or both sets of bars differed from the target temperature by $> \pm 0.5{ }^{\circ} \mathrm{C}$ were discarded. The data from the remaining 44 runs were analysed. The temperatures of bar sets 1 and 2 during the warm run were $42.00{ }^{\circ} \mathrm{C}(\mathrm{SD}=0.30)$ and $41.94{ }^{\circ} \mathrm{C}(0.24)$, respectively. During the cool run, the temperatures of bar sets 1 and 2 were $18.09{ }^{\circ} \mathrm{C}$ $(0.15)$ and $18.17^{\circ} \mathrm{C}(0.11)$.

\subsection{Data analysis}

Data were analysed using SPSS v24. Results were deemed statistically significant for $p<0.05$, two-tailed.

Although the two groups of participants in the main experiment felt different test stimuli, we were able to compare the groups on a common measure of pain sensitivity to the $6{ }^{\circ} \mathrm{C}$ cold pressor stimulus. Here, an independent samples $t$-test comparing the average painfulness of the cold bath across groups was used.

Grill pain intensity and unpleasantness were assessed using separate $2 \times 2$ mixed-model ANOVAs. Here, Group (NH or TG) was a between-subjects factor and Run Type (Control or CPM) was a withinsubjects factor. In the ANOVAs, we assessed (1) the main effect of Group (i.e. whether the painfulness and unpleasantness of the TGI and noxious heat were similar overall), (2) the main effect of Run Type (i.e. whether the conditioning stimulus affected the painfulness of the test stimuli), and (3) the interaction between Group and Run Type (i.e. whether CPM affected the TGI and noxious heat similarly).

In addition to determining the amount of pain intensity and unpleasantness produced by the grills and the amount of CPM-induced reduction in them, we also wished to determine (1) how the grills were perceived qualitatively and (2) how CPM changed the way that the grills were described. Subjects indicated after each run which of nine sensations were experienced from the grill. Since the sensation questionnaires contained categorical (yes/no) responses, non-parametric statistics were used for analysis. Mann-Whitney $U$-tests were conducted to determine whether the proportion that each sensation was endorsed differed between the $\mathrm{NH}$ and TG groups. Whether the frequencies of responses changed significantly between the Control and CPM runs in the main experiment were tested using Wilcoxon signed-rank tests. Each of these tests compared the frequency of responses for one descriptor and one grill type during the Control run to the frequency reported during the CPM run. Finally, Wilcoxon tests were also used to compare differences in the proportion of sensations elicited by the warm and cool stimuli that were applied separately in the component temperatures experiment.

\section{Results}

\subsection{Conditioning stimulus pain ratings}

Subjects gave verbal pain intensity ratings of the water bath at 15, 30 and $45 \mathrm{~s}$ during the conditioning procedure. Pain ratings and water bath temperatures for the neutral (Control run) and the noxious cold (CPM run) water baths are provided in Table 1. Cold pain sensitivity was similar across groups $[t$ $(35)=-0.35 ; p=0.73]$.

\subsection{Grill pain intensity and unpleasantness}

During the Control run (i.e. contralateral hand in neutral water), the $45^{\circ} \mathrm{C}$ bars and the $42{ }^{\circ} \mathrm{C} / 18^{\circ} \mathrm{C}$ bars produced pain intensity ratings of 35.7 $(\mathrm{SD}=25.1)$ and 27.1 (23.9), respectively. During the CPM run (i.e. contralateral hand in cold water), pain intensity of the $\mathrm{NH}$ and $\mathrm{TG}$ grills was reduced by $48.2 \%(M=18.5 ; \mathrm{SD}=20.1)$ and $47.0 \%(M=14.3$; $\mathrm{SD}=13.5$ ), respectively (Fig. $2 \mathrm{~A}$ and $\mathrm{B}$ ). The main effect of Run Type was significant $[F(1,35)=19.8$; $p<0.001]$, indicating a robust reduction in grill pain intensity by CPM. The main effect of Group was not significant $[F(1,35)=1.1 ; p=0.30]$, meaning there was no difference in pain intensity between the hot and warm/cool grills. The interaction between the two factors was not significant $[F(1,35)=0.4$; $p=0.51]$, showing that CPM reduced pain intensity similarly in both groups (Fig. 2C).

Three participants in the TG group did not experience any pain from the TGI (i.e. pain rating $=0$ ). 
Table 1 Water bath temperature and painfulness.

\begin{tabular}{lllcccc}
\hline Condition & Group & Bath Temp $\left({ }^{\circ} \mathrm{C}\right)$ & Rating $1(15 \mathrm{~s})$ & Rating 2 (30 s) & Rating 3 (45 s) & Average bath rating \\
\hline Control & $\mathrm{NH}$ & $32.7(0.2)$ & $1.9(4.7)$ & $1.6(4.6)$ & $1.6(4.7)$ & $1.7(4.6)$ \\
& $\mathrm{TG}$ & $32.6(0.3)$ & $2.2(3.5)$ & $1.9(3.5)$ & $2.0(3.9)$ & $2.1(3.2)$ \\
$\mathrm{CPM}$ & $\mathrm{NH}$ & $6.1(0.1)$ & $38.8(24.6)$ & $52.1(27.0)$ & $62.5(26.5)$ & $51.1(25.1)^{\mathrm{NS}}$ \\
& $\mathrm{TG}$ & $6.1(0.1)$ & $37.2(23.5)$ & $49.4(26.5)$ & $58.1(27.7)$ & $48.2(28.0)^{\mathrm{NS}}$ \\
\hline
\end{tabular}

Note: NH, noxious heat group; TG, thermal grill group; (), standard deviation; NS, difference between groups not significant ( $p=0.73)$.
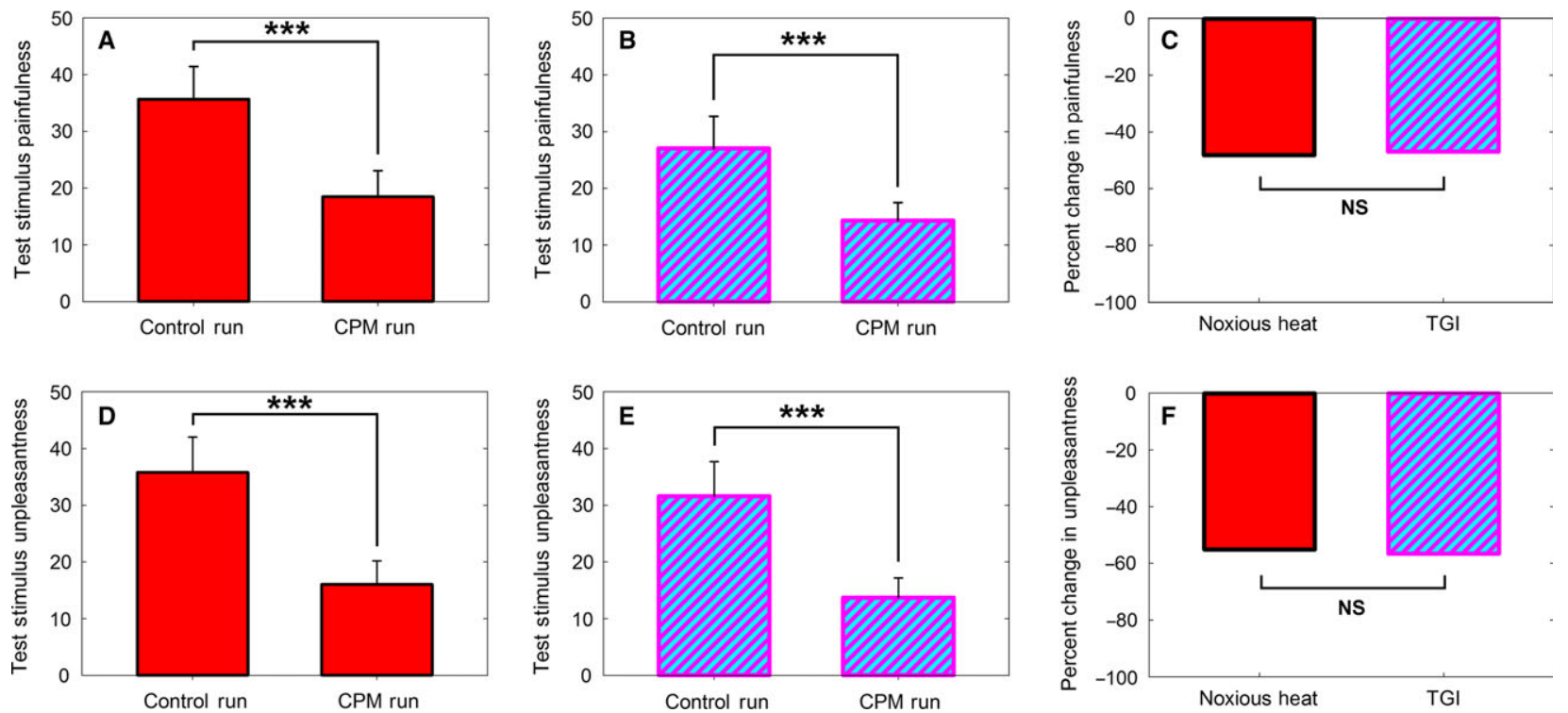

Figure 2 Effects of CPM on pain intensity and unpleasantness. The solid red bars show the results for the noxious heat (NH) group and the striped bars show the thermal grill (TG) group. CPM significantly reduced the painfulness of both (A) noxious heat and (B) the TGI, (C) by nearly $50 \%$ for both types of test stimulation. CPM also reduced the unpleasantness of both (D) noxious heat and (E) the TGl, (F) in these cases by slightly more than $50 \%$. Error bars $=1$ SEM. $* * * p<0.001$. NS, not significant.

The interaction between Group and Run Type was not significant with these three subjects removed $[F$ $(1,32)=0.01 ; p=0.93]$.

$\mathrm{NH}$ unpleasantness $(M=35.8 ; \mathrm{SD}=27.1)$ and $\mathrm{TG}$ unpleasantness $(M=31.6 ; \quad \mathrm{SD}=25.7)$ during the control runs was reduced during CPM by $55.2 \%$ $(M=16.1 ; \quad \mathrm{SD}=18.1) \quad$ and $\quad 56.6 \% \quad(M=13.7$; $\mathrm{SD}=14.6$ ), respectively (Fig. 2D, E and F). The pattern of statistical significance was similar to that for pain intensity. The main effect of Run Type was highly significant $[F(1,35)=26.5 ; p<0.001]$, while the main effect of Group $[F(1,35)=0.3 ; p=0.61]$ and the interaction between the factors $[F$ $(1,35)=0.1 ; p=0.80]$ were not.

One important aspect in the design of our study was to include noxious heat and thermal grill configurations that were perceived to be similarly painful and unpleasant. The lack of main effects of Group reported above indicates that pain intensity and unpleasantness of the two stimuli were similar when considering average test stimulus ratings across both the Control and CPM runs. To further illustrate that differences in the painfulness of the two grill configurations cannot explain the results, post hoc $t$-tests show that the painfulness $[t(35)=1.07, p=0.29]$ and unpleasantness $[t(35)=0.48, p=0.63]$ of the two grill configurations were not different specifically in the Control run, nor were pain $[t(35)=0.73$, $p=0.47]$ and unpleasantness [ $t(35)=0.43, p=0.67]$ of the two grill types different in the CPM runs.

\subsection{Grill descriptors}

The percentages of subjects attributing each of the descriptors to his or her grill experience during the control run are plotted in Fig. 3A. NH subjects most often described their grill as 'hot', followed by 'burning', then 'stinging'. TG subjects most frequently described their grill as 'hot', followed by 'stinging' and 'sharp'. Statistical tests revealed that TG subjects 
significantly more often reported a sensation of 'sharp' than NH subjects $(Z=-2.43 ; p=0.015)$. The frequencies of responses for all other descriptors were not significantly different for the different grill types $(p>0.05$ for all $)$.

Figure $3 \mathrm{~B}$ shows the frequencies of sensations reported for the test stimuli during the CPM run. None of the sensation frequencies significantly differed across the $\mathrm{NH}$ and $\mathrm{TG}$ groups during this run ( $p>0.19$ for all descriptors). Thus, the qualitative aspects of noxious heat and the TGI were also very similar when the processing of these stimuli was being modulated by the noxious conditioning stimulus.

Figure $3 \mathrm{C}$ shows the change in response frequency for the two grills from the Control run to the CPM run. CPM significantly reduced the frequencies of describing noxious heat as 'burning' $(Z=-2.71$; $p=0.007)$ and 'hot' $(Z=-2.53 ; p=0.011)$, while it significantly increased the amount of 'warm' $(Z=-2.45 ; p=0.014)$ responses. For the thermal grill, CPM significantly reduced the number of 'burning' $(Z=-2.24 ; p=0.025)$, 'hot' $(Z=-3.16 ; p=0.002)$ and 'sharp' $(Z=-2.33 ; \quad p=0.02)$ responses; conversely, CPM significantly increased the frequency of 'warm' $(Z=-2.71 ; p=0.007)$ responses.

To summarize, with the exception of the different proportion of 'sharp' responses for the two grills in the Control run, they were very similarly described. Furthermore, CPM changed the perception of the two grills in the same manner: Both grills were less often described as 'burning' and 'hot', and more often indicated as being 'warm', during the CPM compared with the Control run.

\subsubsection{Component temperatures experiment}

The two temperatures comprising the $\mathrm{TG}$ in the main experiment were generally experienced as innocuous when presented individually in the control experiment. No subject reported pain from the $18{ }^{\circ} \mathrm{C}$ bars, and only three described the $42{ }^{\circ} \mathrm{C}$ bars as painful (Fig. 4).

Wilcoxon signed-rank tests showed that the $18{ }^{\circ} \mathrm{C}$ bars were significantly more often described as 'cool' $(Z=-2.31, \quad p=0.02) \quad$ and 'cold' $(Z=3.46$; $p=0.001)$ than the $42{ }^{\circ} \mathrm{C}$ bars, which were more often described as 'warm' $(Z=-2.65 ; p=0.008)$ and 'hot' $(Z=-3.87 ; p<0.001)$. There was no significant difference in the amount of attribution of the remaining descriptors to the cool versus the warm grill $(p>0.05$ for all $)$.

It is important to note that all bars were either warm or cool in this control experiment, as opposed
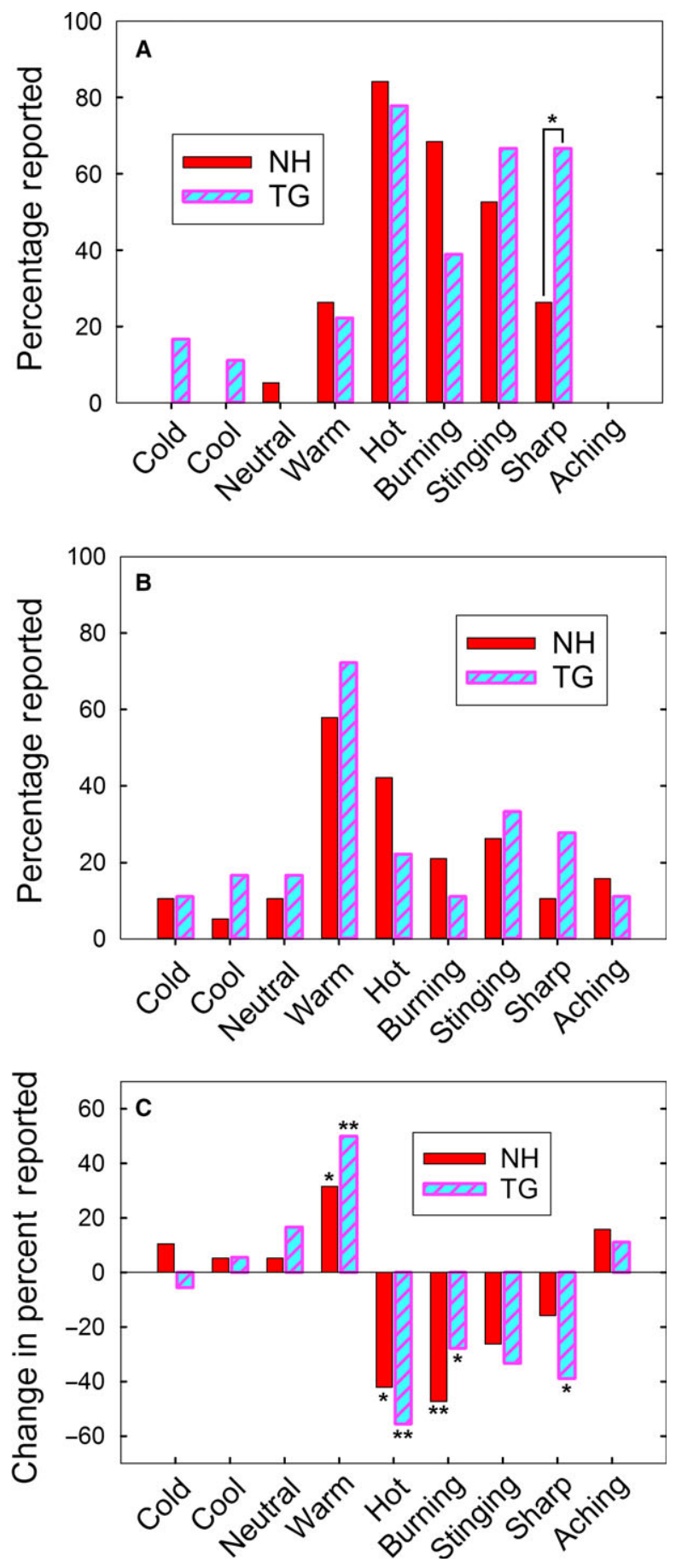

Figure 3 Sensations reported and the effects of CPM on them. (A) Percentage of subjects who reported the indicated sensations in the Control (neutral water bath) run. ${ }^{*} p<0.05$ difference between TG and $\mathrm{NH}$ groups. (B) Percentage of participants who reported the indicated sensations in the CPM run. (C) CPM significantly altered some qualities of the grill stimuli. Each bar represents the percentage that each descriptor was given in the Control run subtracted from the percentage reported during the CPM run. $* p<0.05$ and $* * p<0.01$ difference between Control and CPM runs for a given test stimulus. 


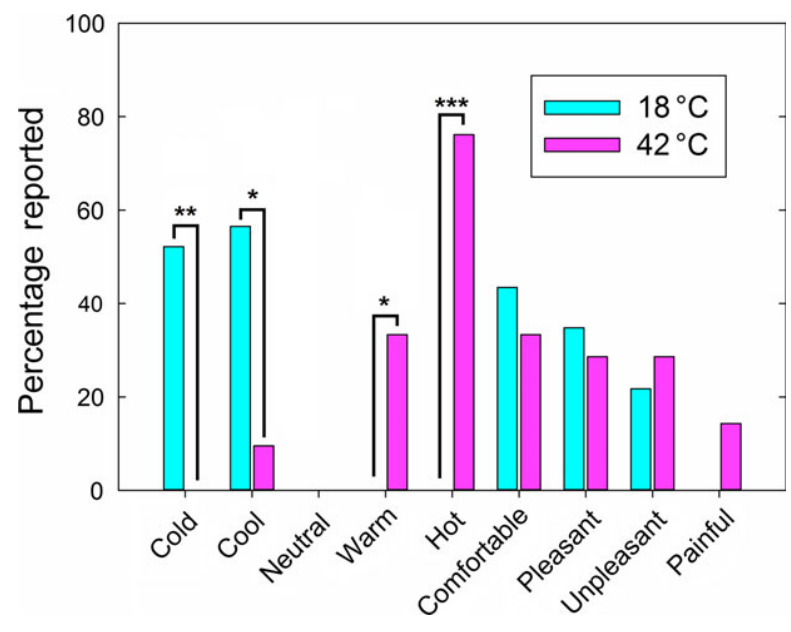

Figure 4 Sensations reported in the component temperatures experiment. The $18{ }^{\circ} \mathrm{C}$ bars (blue) were significantly more often reported to be 'Cool' and 'Cold' than the $42{ }^{\circ} \mathrm{C}$ bars (pink), while the $42{ }^{\circ} \mathrm{C}$ bars were significantly more often called 'Warm' and 'Hot'. There were no other differences in the frequencies of reported sensations between the stimuli. Importantly, each component temperature used to produce the TGI in the main experiment was generally perceived to be innocuous on its own. *p $<0.05 ; * * p<0.01 ; * * p<0.001$.

to just half warm and half cool in the TG stimulus in the main experiment. This likely introduced greater spatial summation within the warm and cool pathways than was present during the main experiment (Hardy and Oppel, 1938; Stevens and Marks, 1971; Defrin et al., 2009), and therefore overstates the perceived intensity of the thermal signals that contributed to the emergence of the thermal grill illusion.

\section{Discussion}

This study reveals that noxious heat $\left(45^{\circ} \mathrm{C}\right)$ and the TGI, which were perceived similarly in terms of their painfulness, unpleasantness and quality, are attenuated to the same extent by CPM.

\subsection{Reductions in pain intensity and unpleasantness}

Our study is not the first to show that the TGI is a graded response that can be modulated within individuals, as the illusion can be attenuated by the administration of analgesics (Kern et al., 2008a,b), by a whole-body thermal challenge (Alfonsi et al., 2016), or by decreasing the temperature differential between the warm and cool component stimuli (Green, 2002; Bouhassira et al., 2005; Adam et al., 2014).

The CPM paradigm is thought to activate DNIC, which attenuates firing in dorsal horn convergent nociceptive neurons (Le Bars et al., 1979). Neuroimaging evidence shows that additional descending inhibitory pathways are activated during CPM in humans (Piche et al., 2009; Sprenger et al., 2011; Bogdanov et al., 2015; Youssef et al., 2016a,b). The degree to which the various mechanisms activated during CPM contribute to reductions in pain is unclear, but descending inhibition operates predominately on nociceptive dorsal horn neurons (Waters and Lumb, 1997; Heinricher et al., 2009; Leith et al., 2010). DNIC does not reduce activity in thermospecific COOL dorsal horn neurons (Dickenson et al., 1980). Electrical stimulation of brainstem regions with descending projections also has similar inhibitory specificity for nociception (Dawson et al., 1981; Davies, 1984), including inhibition of WDR responses to noxious, but not innocuous, cold (Leith et al., 2010). Taken together, these results suggest that descending inhibition does not inhibit ascending innocuous thermal information.

The present results, therefore, seem incompatible with the idea that the TGI percept is a synthesis of innocuous sensations (Alrutz, 1898), but instead suggest the participation of spinal nociceptive neurons, which are subject to descending inhibition.

It should also be noted that CPM can involve cerebral interactions (Sprenger et al., 2011) and be influenced by distraction (Moont et al., 2010) and expectation (Cormier et al., 2013), so some of CPM's inhibition on the test stimuli might have been supraspinal. Since we can be confident that at least some of the CPM-induced reduction in pain was spinally mediated, these results are more consistent with the idea that the TGI is processed in spinal nociceptive neurons; otherwise, its painfulness would have likely been less attenuated by CPM than noxious heat.

\subsection{Nociceptive integration of the TGI}

Craig and Bushnell's (1994) theory posits that TGI pain results from an abnormally high (for innocuous temperatures) ratio of firing in heat/pinch/cold (HPC; i.e. convergent, second-order) to COOL spinal neurons, due to selective inhibition of the latter class by the grill's warm bars. Electrophysiological recordings in cats showed that a cool stimulus caused robust firing in both COOL and HPC cells. Addition of a warm stimulus drastically reduced firing in COOL but not in HPC neurons, leading to a higher HPC/COOL firing ratio. The median HPC threshold for cool stimulation is $24^{\circ} \mathrm{C}$, and their rates systematically increase as temperature is lowered (Craig 
et al., 2001). COOL neurons, in contrast, have thresholds just below normal skin temperature (i.e. $32{ }^{\circ} \mathrm{C}$ ), and they increase firing fairly linearly down to about $15^{\circ} \mathrm{C}$, at which point the response plateaus. Therefore, the HPC/COOL firing ratio starts to increase substantially below $15^{\circ} \mathrm{C}$, approximately cold pain threshold, and could code for the pain of noxious cold. Thus, the TGI could produce pain by mimicking this across-fibre ratio of activity (Craig and Bushnell, 1994). The possibility that cool temperatures evoke nociceptive signals is supported by psychophysical evidence showing that, under certain conditions, mild cooling (ex. $27^{\circ} \mathrm{C}$ ) of the skin can produce sensations of burning and stinging in a majority of subjects (Green, 2002; Green and Pope, 2003). Also, adaptation to the cool, but not the warm, bars of the grill attenuates the TGI's painfulness, further suggesting that the TGI's pain signals are generated by the cool grill temperature (Harper and Hollins, 2014).

In the context of the Craig-Bushnell theory of the TGI, CPM could have attenuated the TGI by increasing COOL cell activity or decreasing HPC activity; either of which would have decreased the HPC/ COOL firing ratio. The first possibility is unlikely, since DNIC does not affect the firing of COOL neurons (Dickenson et al., 1980). Regarding the latter, no study to date has systematically studied the effects of DNIC on HPC neurons, but DNIC is known to inhibit activity in dorsal horn lamina I (Morgan et al., 1994), the location of many HPC neurons (Craig et al., 2001).

Alternatively, TGI pain could be produced by additive, or perhaps even synergistic, responses of innocuous peripheral afferents onto WDR neurons, as posited by Green (2002) and further supported by others (Bouhassira et al., 2005). WDR firing rates in animals across a range of noxious heat intensities correspond very well with psychophysical ratings of perceived pain (Maixner et al., 1986; Coghill et al., 1993). If the grill elicits firing rates in WDR neurons that resemble those produced by noxious heat or cold, this could explain the pain of TGI.

The reductions in TGI pain by CPM observed in this study are also compatible with this theory, since DNIC is well known precisely for its ability to attenuate WDR firing (Le Bars et al., 1979).

\subsection{Qualitative aspects of the TGI}

While the painfulness of the TGI has been questioned (Fruhstorfer et al., 2003; Bach et al., 2011), our results are in agreement with those of many others showing that interlacing warm and cool bars are capable of producing pain (Craig and Bushnell, 1994; Craig et al., 1996; Bouhassira et al., 2005; Leung et al., 2005; Defrin et al., 2008; Kern et al., 2008a,b; Li et al., 2009; Boettger et al., 2011, 2013, 2016; Lindstedt et al., 201la,b). Three subjects (17\%) did not, however, report any TGI pain during the Control run, confirming previous findings of some TGI-insensitive individuals (Bouhassira et al., 2005; Kern et al., 2008a,b; Boettger et al., 2016). Two of these subjects did report 'burning' and 'stinging' and the third reported 'sharp', suggesting that the TGI may still be unpleasant, if not painful, for these individuals.

In addition to reducing the pain intensity and unpleasantness of TGI and nociceptive heat, CPM also reduced the frequency of pain-related adjectives assigned to the two stimuli. 'Burning' was significantly less frequently reported in both groups and 'sharp' was less often attributed to the TGI during CPM. Thus, the comparable reductions in TG and $\mathrm{NH}$ pain by CPM are paralleled by similar changes in the pain-related qualities assigned to them.

The effect of CPM on the perceived thermal intensity of both grills lends further credence to the idea that TGI is generated by a pattern of neural activity closely resembling that elicited by noxious heat. In both groups, the frequency of 'hot' responses was significantly reduced during CPM and the most frequent response became 'warm'. Given that descending inhibition selectively attenuates nociceptive signals (Dickenson et al., 1980; Dawson et al., 1981; Davies, 1984; Leith et al., 2010), CPM's effect implies that nociceptive signals participate in coding the perceived thermal intensity of hot temperatures, even under normal (i.e. noxious heat) conditions.

\subsection{Limitations}

This study has several limitations that should be noted. First, we chose to use a between-subject design for the two grill configurations, in order to eliminate order effects such as bias, adaptation, or sensitization; however, we cannot rule out the possibility that individual differences between groups affected the results in some way. Similarly, the main and component temperatures experiments were carried out on different groups of subjects; although drawn from the same population, it is not certain that the pain and thermal sensitivities of these groups were equivalent.

A second limitation follows from the fact that the CPM procedure can engage multiple endogenous 
mechanisms to reduce pain. It is, therefore, theoretically possible that the painfulness of noxious heat was reduced primarily via one of CPM's underlying mechanisms and the TGI's painfulness through another, despite the close comparability of CPM's effects on the two test stimuli.

Another issue, raised by a reviewer, is that the cold pressor can slightly lower skin temperature of the contralateral limb (Chwalczynska et al., 2015); however, the potential cooling $\left(<1{ }^{\circ} \mathrm{C}\right)$ is too small to explain the robust reductions in pain we observed or to significantly alter thermal sensation (Croze et al., 1977; Strigo et al., 2000).

Finally, in the component temperatures experiment, we warmed or cooled all 12 bars to control for the total area of stimulation (i.e. either warm plus cool or all hot) in the main experiment; we did not include a condition equating the amount of warm or cool stimulation in the main experiment's TG condition by cooling or warming only 6 bars at a time.

\section{Conclusions}

In summary, this study shows that the TGI and noxious heat are attenuated by CPM to the same extent. Based on known CPM mechanisms, this is consistent with TGI theories suggesting that signals from the warm/cool grill give rise to activity in dorsal horn convergent neurons, which generate the 'illusory' pain of the TGI.

\section{Acknowledgements}

The authors thank John Irvin for his involvement in planning and conducting this study and Jade Poteat for her help in collecting data.

\section{Author contributions}

DEH was involved with conception, design, data acquisition, analysis and interpretation, and he drafted the manuscript. MH was involved with conception, design, data analysis, and interpretation, and he critically revised the manuscript. Both authors discussed the results and gave their approval of the final manuscript.

\section{References}

Adam, F., Alfonsi, P., Kern, D., Bouhassira, D. (2014). Relationships between the paradoxical painful and nonpainful sensations induced by a thermal grill. Pain 155, 2612-2617.

Alfonsi, P., Adam, F., Bouhassira, D. (2016). Thermoregulation and pain perception: Evidence for a homoeostatic (interoceptive) dimension of pain. Eur J Pain 20, 138-148.
Alrutz, S. (1898). On the temperature senses: II. The sensation 'hot'. Mind 7, 141-144.

Bach, P., Becker, S., Kleinbohl, D., Holzl, R. (2011). The thermal grill illusion and what is painful about it. Neurosci Lett 505, 31-35.

Boettger, M.K., Schwier, C., Bar, K.J. (2011). Sad mood increases pain sensitivity upon thermal grill illusion stimulation: Implications for central pain processing. Pain 152, 123-130.

Boettger, M.K., Grossmann, D., Bar, K.J. (2013). Thresholds and perception of cold pain, heat pain, and the thermal grill illusion in patients with major depressive disorder. Psychosom Med 75, 281-287.

Boettger, M.K., Ditze, G., Bar, K.J., Krudewagen, E.M., Schaible, H.G. (2016). Humans, but not animals, perceive the thermal grill illusion as painful. Behav Brain Res 313, 172-176.

Bogdanov, V.B., Vigano, A., Noirhomme, Q., Bogdanova, O.V., Guy, N. et al. (2015). Cerebral responses and role of the prefrontal cortex in conditioned pain modulation: An fMRI study in healthy subjects. Behav Brain Res 281, 187-198.

Bouhassira, D., Kern, D., Rouaud, J., Pelle-Lancien, E., Morain, F. (2005). Investigation of the paradoxical painful sensation ('illusion of pain') produced by a thermal grill. Pain 114, 160-167.

Chwalczynska, A., Gruszka, K., Calkosinski, I., Sobiech, K.A. (2015). Thermovision analysis changes of human hand surface temperature in cold pressor test. Biomed Res Int 2015, 783642.

Coghill, R.C., Mayer, D.J., Price, D.D. (1993). Wide dynamic range but not nociceptive-specific neurons encode multidimensional features of prolonged repetitive heat pain. J Neurophysiol 69, 703-716.

Cormier, S., Piche, M., Rainville, P. (2013). Expectations modulate heterotopic noxious counter-stimulation analgesia. J Pain 14, 114 125.

Craig, A.D., Bushnell, M.C. (1994). The thermal grill illusion: Unmasking the burn of cold pain. Science 265, 252-255.

Craig, A.D., Reiman, E.M., Evans, A., Bushnell, M.C. (1996). Functional imaging of an illusion of pain. Nature 384, 258-260.

Craig, A.D., Krout, K., Andrew, D. (2001). Quantitative response characteristics of thermoreceptive and nociceptive lamina I spinothalamic neurons in the cat. J Neurophysiol 86, 1459-1480.

Croze, S., Duclaux, R., Russek, M. (1977). Constancy of heat pain characteristics to changes in skin and body temperature. Brain Res 131, 367-372.

Davies, S.N. (1984). Evidence for peripheral, but not central modulation of trigeminal cold receptive cells in the rat. Brain Res 301, 299-305.

Dawson, N.J., Dickenson, A.H., Hellon, R.F., Woolf, C.J. (1981). Inhibitory controls on thermal neurones in the spinal trigeminal nucleus of cats and rats. Brain Res 209, 440-445.

Defrin, R., Benstein-Sheraizin, A., Bezalel, A., Mantzur, O., ArendtNielsen, L. (2008). The spatial characteristics of the painful thermal grill illusion. Pain 138, 577-586.

Defrin, R., Petrini, L., Arendt-Nielsen, L. (2009). Spatial summation of thermal sensations depends on skin type and skin sensitivity. Exp Brain Res 198, 29-36.

Dickenson, A.H., Le Bars, D., Besson, J.M. (1980). Diffuse noxious inhibitory controls (DNIC). Effects on trigeminal nucleus caudalis neurones in the rat. Brain Res 200, 293-305.

Fruhstorfer, H., Harju, E.L., Lindblom, U.F. (2003). The significance of A-delta and C fibres for the perception of synthetic heat. Eur J Pain 7, 63-71.

Granot, M., Weissman-Fogel, I., Crispel, Y., Pud, D., Granovsky, Y., Sprecher, E., Yarnitsky, D. (2008). Determinants of endogenous analgesia magnitude in a diffuse noxious inhibitory control (DNIC) paradigm: Do conditioning stimulus painfulness, gender and personality variables matter? Pain 136, 142-149.

Green, B.G. (2002). Synthetic heat at mild temperatures. Somatosens Mot Res 19, 130-138.

Green, B.G., Pope, J.V. (2003). Innocuous cooling can produce nociceptive sensations that are inhibited during dynamic mechanical contact. Exp Brain Res 148, 290-299.

Hardy, J.D., Oppel, T.W. (1938). Studies in temperature sensation. IV. The stimulation of cold sensation by radiation. J Clin Invest 17, 771778 . 
Harper, D.E., Hollins, M. (2014). Coolness both underlies and protects against the painfulness of the thermal grill illusion. Pain 155, 801-807.

Heinricher, M.M., Tavares, I., Leith, J.L., Lumb, B.M. (2009). Descending control of nociception: Specificity, recruitment and plasticity. Brain Res Rev 60, 214-225.

Kern, D., Pelle-Lancien, E., Luce, V., Bouhassira, D. (2008a). Pharmacological dissection of the paradoxical pain induced by a thermal grill. Pain 135, 291-299.

Kern, D., Plantevin, F., Bouhassira, D. (2008b). Effects of morphine on the experimental illusion of pain produced by a thermal grill. Pain $139,653-659$

Le Bars, D., Dickenson, A.H., Besson, J.M. (1979). Diffuse noxious inhibitory controls (DNIC). I. Effects on dorsal horn convergent neurones in the rat. Pain 6, 283-304.

Leith, J.L., Koutsikou, S., Lumb, B.M., Apps, R. (2010). Spinal processing of noxious and innocuous cold information: Differential modulation by the periaqueductal gray. J Neurosci 30, 4933-4942.

Leung, A.Y., Wallace, M.S., Schulteis, G., Yaksh, T.L. (2005). Qualitative and quantitative characterization of the thermal grill. Pain $116,26-32$.

Li, X., Petrini, L., Wang, L., Defrin, R., Arendt-Nielsen, L. (2009). The importance of stimulus parameters for the experience of the thermal grill illusion. Neurophysiol Clin 39, 275-282.

Lindstedt, F., Johansson, B., Martinsen, S., Kosek, E., Fransson, P., Ingvar, M. (2011a). Evidence for thalamic involvement in the thermal grill illusion: An FMRI study. PLOS ONE 6, e27075.

Lindstedt, F., Lonsdorf, T.B., Schalling, M., Kosek, E., Ingvar, M. (2011b). Perception of thermal pain and the thermal grill illusion is associated with polymorphisms in the serotonin transporter gene. PLOS ONE 6, el7752.

Maixner, W., Dubner, R., Bushnell, M.C., Kenshalo, D.R., Oliveras, J.L. (1986). Wide-dynamic-range dorsal horn neurons participate in the encoding process by which monkeys perceive the intensity of noxious heat stimuli. Brain Res 374, 385-388.

Moont, R., Pud, D., Sprecher, E., Sharvit, G., Yarnitsky, D. (2010). 'Pain inhibits pain' mechanisms: Is pain modulation simply due to distraction? Pain 150, 113-120.
Morgan, M.M., Gogas, K.R., Basbaum, A.I. (1994). Diffuse noxious inhibitory controls reduce the expression of noxious stimulus-evoked Fos-like immunoreactivity in the superficial and deep laminae of the rat spinal cord. Pain 56, 347-352.

Nir, R.R., Granovsky, Y., Yarnitsky, D., Sprecher, E., Granot, M. (2011). A psychophysical study of endogenous analgesia: The role of the conditioning pain in the induction and magnitude of conditioned pain modulation. Eur J Pain 15, 491-497.

Piche, M., Arsenault, M., Rainville, P. (2009). Cerebral and cerebrospinal processes underlying counterirritation analgesia. J Neurosci 29, 14236-14246.

Sprenger, C., Bingel, U., Buchel, C. (2011). Treating pain with pain: Supraspinal mechanisms of endogenous analgesia elicited by heterotopic noxious conditioning stimulation. Pain 152, 428-439.

Stevens, J.C., Marks, L.E. (1971). Spatial summation and the dynamics of warmth sensation. Percept Psychophys 9, 291-298.

Strigo, I.A., Carli, F., Bushnell, M.C. (2000). Effect of ambient temperature on human pain and temperature perception. Anesthesiology 92, 699-707.

Thunberg, T. (1896). Förnimmelserna vid till samma ställe lokasirerad, samtidigtb pagaende köld och värmeretning. Uppsala Läkkfören Föhr 489-495.

Waters, A.J., Lumb, B.M. (1997). Inhibitory effects evoked from both the lateral and ventrolateral periaqueductal grey are selective for the nociceptive responses of rat dorsal horn neurones. Brain Res 752, 239-249.

Willer, J.C., Roby, A., Le Bars, D. (1984). Psychophysical and electrophysiological approaches to the pain-relieving effects of heterotopic nociceptive stimuli. Brain 107(Pt 4), 1095-1112.

Youssef, A.M., Macefield, V.G., Henderson, L.A. (2016a). Cortical influences on brainstem circuitry responsible for conditioned pain modulation in humans. Hum Brain Mapp 37, 2630-2644.

Youssef, A.M., Macefield, V.G., Henderson, L.A. (2016b). Pain inhibits pain; human brainstem mechanisms. NeuroImage 124, 54-62. 